\title{
Assessing the Effect of Different Agroforestry Practices on Soil Physico-chemical Properties and Microbial Activity
}

\author{
Baswant Sushant Pralhad $^{1 *}$, P. Rajendran ${ }^{1}$, M. P. Divya ${ }^{2}$, \\ R. Rajeswari $^{3}$, G. Thangamani ${ }^{1}$ and C. Ramaha $^{1}$ \\ ${ }^{1}$ Department of Agroforestry, ${ }^{2}$ Department of Forest Product and Wildlife, ${ }^{3}$ Department of \\ Sericulture, Forest College \& Research Institute, Mettupalayam, India \\ *Corresponding author
}

\section{A B S T R A C T}

\begin{tabular}{l} 
K e y w o r d s \\
Agroforestry, \\
Bacteria, Fungi, \\
Actinomycetes, \\
Greengram, \\
Blackgram, \\
Cowpea, Gardenpea \\
\hline Article Info \\
\hline $\begin{array}{l}\text { Accepted: } \\
18 \text { November } 2020 \\
\text { Available Online: } \\
\text { 10 December } 2020\end{array}$ \\
\hline
\end{tabular}

Keywords

Agroforestry, Bacteria, Fungi, Actinomycetes,

Blackgram,

Cowpea, Gardenpea

\section{Introduction}

Agroforestry refers to land use systems and technologies in which woody perennials are deliberately combined on the same land management unit with agricultural crops and/or animals in some form of spatial arrangement or temporal sequence (Nair, 1984; 1989). Although a plethora of benefits such as nutrient pumping from deeper soil profiles, fixation of atmospheric nitrogen, reduction of soil evaporation, mitigation of soil erosion, increase of soil nutrient status, improvement of soil structure (Nair, 1984) have been attributed to this land use system, these are mostly assumptions based on indirect evidence and only a few of these benefits have been established on the basis of scientific knowledge. In fact (Young, 1989a) proposed ten general hypotheses that agroforestry system in general and trees in particular can conserve or improve soil fertility, Subsequently, an eleventh hypothesis relating to influence of tree canopies on soil 
fertility (Willison, 1990)and a twelfth hypothesis that roots of $\mathrm{N}_{2}$ fixing trees possess more nodules when in intimacy with roots of non- $\mathrm{N}_{2}$ fixing trees have also been propounded. While logically an agroforestry system may be more conservation effective than an arable land use system, there are few quantitative measurements to prove the validity of such a logic (Lal, 1989).

Healthy soil is one of the most critical resources for the health and sustainability of ecosystems, including agroecosystems. Agroforestry, as a sustainable land management practice, has shown solid evidence of its role in improving soil quality and health based on four decades of data gathered from the world over. Agroforestry has the ability to (1) enrich soil organic carbon (SOC) better than mono-cropping systems, (2) improve soil nutrient availability and soil fertility due to the presence of trees in the system, and (3) enhance soil microbial dynamics, which would positively influence soil health (Dollinger and Jose, 2018).

The importance of tree based land use system is that it keeps in restoring soil fertility and improving the economy of farmers having small land holdings (Dhyani and Chauhan, 1990). Improvement in soil fertility under agroforestry systems occurs mainly through addition of the both crop and tree components. The potential of agroforestry to improve soil quality has been widely recognized as a major benefit since its inception as a scientifically recognized discipline and practice (Young, 1989b; Nair, 2011).

Perennial woody tree species are the important component of agroforestry systems. They reduce nutrient losses from the productive system through efficient nutrient cycling. Addition of nutrients through litter decomposition, dead root biomass and $\mathrm{N}_{2}$ fixation increases importance of tree species in soil nutrient status improvement.

Palani (1996) reported that when compared to pure crops, the soil fertility status under the agroforestry system has been improved. The soil properties of various tree species have been studied by (Yadav, 2008). In agroforestry systems, the SOC was higher. The increase in the fertility of the soil was due to a fall in litter. Positive changes in different physical, chemical and biological soil indicators in the 10-year age group $A$. Procera-based AF processes (Kikon et al., 2017). The growth characteristics of selected tree species, viz., Leucaena leucocephala, Grewia optiva, Albizzia lebbek based agricultural systems, were examined by(Gupta et al., 2017). Physical and chemical properties of both the surface $(0$ to $15 \mathrm{~cm})$ and the sub-surface $(15$ to $30 \mathrm{~cm}$ ) layer of the soil under the agricultural systems below. The minimum bulk density (1.21 and $1.28 \mathrm{~g} \mathrm{~cm}^{-3}$ ) was determined under $A$. Lebbek, and highest underG. optiva (1.26 and $\left.1.31 \mathrm{~g} \mathrm{~cm}^{-3}\right)$.

SOC and microbial population studies often focus on the upper $20-30 \mathrm{~cm}$ of soil, as this is considered the peak percentage of the soil contour that is biologically vigorous(Jobbágy and Jackson, 2000; Veldkamp et al., 2003; Goberna et al., 2006; Baisden and Parfitt, 2007). Portella et al., (2012) discovered that under agroforestry systems, fresh organic matter acts as an ideal medium for increasing microbial activity due to continuous accumulation of fresh organic matter, which acts as a source for increasing the strength of the other aggregates and maintaining well pore distribution. Meshram et al., (2016)found that the maximum bacterial population (26.28 CFUX $\left.10^{7} \mathrm{~g}\right)$ was registered under $A$. mangium, the maximum population of fungal (15.90 CFU X $10^{4} \mathrm{~g}$ ) was recorded under $A$. holosericea and the maximum population $\left(14.27 \mathrm{CFU} \mathrm{X} 10^{6} \mathrm{~g}\right)$ of 
actinomycetes under $G$. sepium in ten different $\mathrm{N}$ fixing tree species viz.,Cassia siamea, Acacia catechu, Acacia mangium, Gliricidia sepium, Pterocarpus marsupium, Pterocarpus marsupium, Acacia holosericea, Acacia auriculiformis, Albizzia lebbeck, Casuarina equisetifolia and Dalbergia sissoo.

The experiment was designed to analyse the effects of different agroforestry practices on soil physico-chemical properties and microbial community and to compare the properties before and after the experiment.

\section{Materials and Methods}

The Field experiment was conducted during 2019-20 at Forest College and Research Institute, Mettupalayam. The total area encompasses 200 ha, under sylvan forest range, $40 \mathrm{~km}$ away from Coimbatore in the northern direction at the foothills of the Nilgiris lying between $11^{\circ} 19^{\prime}$ and $11^{\circ} 20^{\prime} \mathrm{N}$ latitude, $76^{\circ} 56^{\prime}$ and $76^{\circ} 57^{\prime} \mathrm{E}$ longitude and at an altitude of $300 \mathrm{~m}$ above MSL. The present experiment was framed in Randomized Block Design (RBD) with thirty treatments and two replications in different 5 years old trees under agroforestry practices. The block plantation tree species comprises viz., Khaya senegalensis, Melia dubia, Dalbergia sissoo, Populus deltoides, Casuarina equositifolia and Control (Open area) with four different intercrops viz., greengram, cowpea, blackgram, garden pea. Trees species we are planted in 5 x $5 \mathrm{~m}$ spacing.

A soil samples were collected from under each tree-crop combinations at two different depths0-30 $\mathrm{cm}$ and $30-60 \mathrm{~cm}$ at approximately $1 \mathrm{~m}$ away from the tree base and for microbial analysis soil samples were collected from under each tree-crop combinations at two different depths $0-15 \mathrm{~cm}$ and $15-30 \mathrm{~cm}$. Each species such sampling was done at two locations sufficiently removed from one another which served as replications. Soil samples were also collected from the open area at two depths and two locations. Collected soil samples were air dried, powdered and passing through $<2 \mathrm{~mm}$ sieve for determination of physico-chemical properties. Organic carbon was determined by (Walkley and Black, 1934) wet oxidation method $\mathrm{pH}$ and Electrical conductivity of soil water saturated pastes were measured by using $\mathrm{pH}$ meter and conductivity meter (Jackson, 1973). Available soil nitrogen was determined by alkaline- $\mathrm{KMnO}_{4}$ methods (Subbiah and Asija, 1956). Available phosphorus was determined by Olsen's method (Olsen, 1954). Available potassium is determined by flame photometric method suggested by(Stanford and English, 1949). For microbial populations (bacteria, fungi and actinomycetes) were determined by serial dilution plate techniques method was given by (Parkinson et al., 1971).

The experimental data was subjected to analysis of variances (ANOVA) and treatment means were compared, significant differences were tested at $\mathrm{p}=0.05$ using randomized block design (RBD) as given by (Panse and Sukhatme, 1954).

\section{Results and Discussion}

Soil samples of both initial and post-harvest were taken from five different agroforestry practices and analyzed for $\mathrm{pH}, \mathrm{EC}$, organic carbon, available $\mathrm{N}, \mathrm{P}$, and $\mathrm{K}$ as per the standard procedure (Fig. 1-9 and Table 1-4).

\section{Soil pH}

Data with respect to the effect of intercropping on soil $\mathrm{pH}$ is presented in (Table 2). The soil $\mathrm{pH}$ values under intercrops and tree alone treatment after harvesting of intercrops were slightly decreased from the initial soil samples but the differences among 
the treatment were meager. However, highest $\mathrm{pH}$ value of surface and sub-surface soil recorded under Melia dubia +cowpea combination treatment $\left(\mathrm{T}_{8}\right)$ (8.14 and 8.01), and the lowest values were recorded under Khaya senegalensis + blackgram combination $\left(\mathrm{T}_{4}\right)$ of surface and sub-surface soil (7.62 and 7.59) (Table 2). Similar result reported that the soil $\mathrm{p}^{\mathrm{H}}$ was significantly lower compared to sole cropping under turmeric intercropped with bamboo based AF systems (Kikon et al., 2017). The minimum value $\mathrm{p}^{\mathrm{H}}$ was recorded under G. optiva (6.47 and 6.59) and maximum in L. leucocephala (6.58 and 6.67) under agrisilvicultural systems (Gupta et al., 2017).Singh et al., (2018) reported that initial $\mathrm{pH}$ of soil was found slightly alkaline with the $\mathrm{pH} \quad(8.20$ and 8.18) it was decreased continuous cropping of four years with the $\mathrm{pH}$ value of near neutral (7.82) under agroforestry systems.

\section{Soil Electrical Conductivity (EC)}

Data pertaining to the effect of intercrops on soil EC is presented in (Table 2). The intercrops have slightly increased the soil EC but the differences among the treatment were meager. However, highest EC value recorded under Populus deltoids trees alone $\left(\mathrm{T}_{16}\right)$ of surface and sub-surface soil $(0.21$ and 0.25 $\left.\mathrm{dSm}^{-1}\right)$. The lowest EC value was noticed under open area (Barren land) treatment $\left(\mathrm{T}_{30}\right)$ of surface and sub-surface soil $(0.14$ and $0.15 \mathrm{dSm}^{-1}$ ) (Table 2). Meshram et al., (2016) found, the EC value was varied from 0.10 to $0.27 \mathrm{dSm}^{-1}$ and maximum EC was recorded under A. mangium.

In contrast to without applying fertilizers, the Soil EC decreased with intercropping of Tectona grandis with groundnut, whereas existing nitrogen, phosphorus and potassium increased (Mutanal et al., 2000). The increase in soil EC might be due to the addition of fertilizers added both for tree and intercrops.

\section{Soil Organic Carbon (SOC)}

Data with regard to the effect of intercrops on soil organic carbon is presented in (Table 2). There was a little build-up of organic carbon in the soil after intercropping under all the treatments including tree alone treatment when compared to initial value. The highest amount of organic carbon of surface and subsurface soil was observed under Melia dubia+ cowpea combination ( $\left.\mathrm{T}_{8}\right)$ (0.95-0.94\%)and the lowest organic carbon content of surface and sub-surface soil was observed in Khaya senegalensis tree alone $\left(\mathrm{T}_{1}\right)(0.76$ and $0.80 \%)$ treatment (Table 2). Similar result found that chemical properties viz., OC, available nitrogen, available phosphorus, available potassium were recorded significantly higher in surface layer ( 0 to $15 \mathrm{~cm}$ ) which decreased significantly with successively increase in soil depth (Sharma et al., 2016). Prasad et al., (2015) observed that SOC build up agroforestry plots over pure crop must have been caused by addition of slowly decomposable carbon in the form of leaf litter and root in biomass of trees. Singh et al., (2018)the organic carbon $(0.59 \%)$ of soil was increased due to crop residues and leaves fall from tree and also intercropping pulse (fixing nitrogen from the atmosphere) also adding the organic matter to the soil under Melia composite and Emblica officinalis based agri silvi medicinal agroforestry system. The highest amount of OC was observed under red gram ( $7.00 \mathrm{~g} \mathrm{C} \mathrm{kg}^{-1}$ ) followed by green gram (6.90 $\mathrm{g} \mathrm{C} \mathrm{kg}^{-1}$ ) treatments and the lowest organic carbon content was observed in tree alone (6.6 $\mathrm{g} \mathrm{C} \mathrm{kg}^{-1}$ ) treatment (Selvam, 2011).

\section{Soil available nitrogen}

Effect of intercrops on soil available nitrogen is presented in (Table 3). Among the intercrops tried the higher available $\mathrm{N}$ of surface and sub-surface soil was found in Khaya senegalensis+ blackgram combination 
$\left(\mathrm{T}_{4}\right)\left(355.1\right.$ and $\left.372.19 \mathrm{~kg} \mathrm{ha}^{-1}\right)$ and lowest was in Populus deltoids + gardenpea combination $\left(\mathrm{T}_{20}\right)\left(203.87\right.$ and $\left.212.99 \mathrm{~kg} \mathrm{ha}^{-1}\right)$ (Table 3). Kikon et al., (2017) reported that soil $\mathrm{p}^{\mathrm{H}}$, EC and $\mathrm{BD}$ were significantly lower and available nitrogen, phosphorus and potassium were significantly higher compared to sole cropping under turmeric intercropped with bamboo based AF systems. The chemical properties viz., OC, available nitrogen, available phosphorus, available potassium were recorded significantly higher in surface layer $(0$ to $15 \mathrm{~cm})$ which decreased significantly with successively increase in soil depth (Sharma et al., 2016).

Table.1 Treatment details of different agroforestry practices

\begin{tabular}{|c|l|}
\hline Sl. No. & \multicolumn{1}{|c|}{ Treatment } \\
\hline $\mathbf{1}$ & $\mathrm{T}_{1}$ Khaya senegalensis trees alone \\
\hline $\mathbf{2}$ & $\mathrm{T}_{2}$ Khaya senegalensis + Greengram combination \\
\hline $\mathbf{3}$ & $\mathrm{T}_{3}$ Khaya senegalensis + Cowpea combination \\
\hline $\mathbf{4}$ & $\mathrm{T}_{4}$ Khaya senegalensis + Blackgram combination \\
\hline $\mathbf{5}$ & $\mathrm{T}_{5}$ Khaya senegalensis + Gardenpea combination \\
\hline $\mathbf{6}$ & $\mathrm{T}_{6}$ Melia dubia trees alone \\
\hline $\mathbf{7}$ & $\mathrm{T}_{7}$ Melia dubia + Greengram combination \\
\hline $\mathbf{8}$ & $\mathrm{T}_{8}$ Melia dubia + Cowpea combination \\
\hline $\mathbf{9}$ & $\mathrm{T}_{9}$ Melia dubia + Blackgram combination \\
\hline $\mathbf{1 0}$ & $\mathrm{T}_{10}$ Melia dubia + Gardenpea combination \\
\hline $\mathbf{1 1}$ & $\mathrm{T}_{11}$ Dalbergia sissoo trees alone \\
\hline $\mathbf{1 2}$ & $\mathrm{T}_{12}$ Dalbergia sissoo + Greengram combination \\
\hline $\mathbf{1 3}$ & $\mathrm{T}_{13}$ Dalbergia sissoo + Cowpea combination \\
\hline $\mathbf{1 4}$ & $\mathrm{T}_{14}$ Dalbergia sissoo + Blackgram combination \\
\hline $\mathbf{1 5}$ & $\mathrm{T}_{15}$ Dalbergia sissoo + Gardenpea combination \\
\hline $\mathbf{1 6}$ & $\mathrm{T}_{16}$ Populus deltoides trees alone \\
\hline $\mathbf{1 7}$ & $\mathrm{T}_{17}$ Populus deltoides + Greengram combination \\
\hline $\mathbf{1 8}$ & $\mathrm{T}_{18}$ Populus deltoides + Cowpea combination \\
\hline $\mathbf{1 9}$ & $\mathrm{T}_{19}$ Populus deltoides + Blackgram combination \\
\hline $\mathbf{2 0}$ & $\mathrm{T}_{20}$ Populus deltoides + Gardenpea combination \\
\hline $\mathbf{2 1}$ & $\mathrm{T}_{21}$ Casuarina equositifolia trees alone \\
\hline $\mathbf{2 2}$ & $\mathrm{T}_{22}$ Casuarina equositifolia + Greengram combination \\
\hline $\mathbf{2 3}$ & $\mathrm{T}_{23}$ Casuarina equositifolia + Cowpea combination \\
\hline $\mathbf{2 4}$ & $\mathrm{T}_{24}$ Casuarina equositifolia + Blackgram combination \\
\hline $\mathbf{2 5}$ & $\mathrm{T}_{25}$ Casuarina equositifolia + Gardenpea combination \\
\hline $\mathbf{2 6}$ & $\mathrm{T}_{26}$ Greengram alone \\
\hline $\mathbf{2 7}$ & $\mathrm{T}_{27}$ Cowpea alone \\
\hline $\mathbf{2 8}$ & $\mathrm{T}_{28}$ Blackgram alone \\
\hline $\mathbf{2 9}$ & $\mathrm{T}_{29}$ Gardenpea alone \\
\hline $\mathbf{3 0}$ & $\mathrm{T}_{30}$ Openarea (Barren area) \\
\hline & \\
\hline
\end{tabular}


Table.2 Soil pH, EC and OC under different agroforestry practices

\begin{tabular}{|c|c|c|c|c|c|c|c|c|c|c|c|c|c|c|c|c|c|c|}
\hline \multirow[t]{3}{*}{ Treatment } & \multicolumn{6}{|c|}{ Soil pH } & \multicolumn{6}{|c|}{ Soil EC } & \multicolumn{6}{|c|}{ Soil OC } \\
\hline & \multicolumn{3}{|c|}{ Before } & \multicolumn{3}{|c|}{ After } & \multicolumn{3}{|c|}{ Before } & \multicolumn{3}{|c|}{ After } & \multicolumn{3}{|c|}{ Before } & \multicolumn{3}{|c|}{ After } \\
\hline & 30 & 60 & Mean & 30 & 60 & Mean & 30 & 60 & Mean & 30 & 60 & Mean & 30 & 60 & Mean & 30 & 60 & Mean \\
\hline T1 & 7.95 & 7.89 & 7.92 & 7.93 & 7.86 & 7.89 & 0.19 & 0.18 & 0.19 & 0.21 & 0.18 & 0.20 & 0.79 & 0.73 & 0.76 & 0.81 & 0.78 & 0.80 \\
\hline $\mathbf{T} 2$ & 7.91 & 7.83 & 7.87 & 7.87 & 7.81 & 7.84 & 0.20 & 0.16 & 0.18 & 0.23 & 0.21 & 0.22 & 0.89 & 0.94 & 0.92 & 0.92 & 0.96 & 0.94 \\
\hline T3 & 7.97 & 7.87 & 7.92 & 7.95 & 7.83 & 7.89 & 0.18 & 0.15 & 0.17 & 0.22 & 0.17 & 0.20 & 0.84 & 0.79 & 0.82 & 0.86 & 0.81 & 0.84 \\
\hline T4 & 7.64 & 7.61 & 7.62 & 7.61 & 7.57 & 7.59 & 0.21 & 0.15 & 0.18 & 0.23 & 0.18 & 0.21 & 0.96 & 0.88 & 0.82 & 0.99 & 0.89 & 0.94 \\
\hline T5 & 7.99 & 7.86 & 7.93 & 7.89 & 7.78 & 7.83 & 0.19 & 0.14 & 0.17 & 0.21 & 0.19 & 0.20 & 0.95 & 0.92 & 0.94 & 0.96 & 0.94 & 0.95 \\
\hline T6 & 8.07 & 7.91 & 7.99 & 7.97 & 7.83 & 7.90 & 0.18 & 0.15 & 0.17 & 0.20 & 0.17 & 0.19 & 0.91 & 0.94 & 0.93 & 0.93 & 0.94 & 0.93 \\
\hline $\mathbf{T 7}$ & 8.15 & 8.01 & 8.08 & 8.01 & 7.95 & 7.98 & 0.20 & 0.16 & 0.18 & 0.22 & 0.18 & 0.20 & 0.94 & 0.90 & 0.92 & 0.97 & 0.93 & 0.95 \\
\hline T8 & 8.17 & 8.11 & 8.14 & 8.03 & 7.99 & 8.01 & 0.17 & 0.15 & 0.16 & 0.21 & 0.16 & 0.19 & 0.94 & 0.95 & 0.95 & 0.99 & 0.89 & 0.94 \\
\hline T9 & 8.12 & 8.06 & 8.09 & 7.96 & 7.89 & 7.91 & 0.19 & 0.16 & 0.18 & 0.21 & 0.18 & 0.20 & 0.89 & 0.86 & 0.88 & 0.96 & 0.94 & 0.95 \\
\hline T10 & 8.03 & 7.98 & 8.01 & 7.98 & 7.93 & 7.95 & 0.19 & 0.14 & 0.17 & 0.24 & 0.21 & 0.23 & 0.89 & 0.93 & 0.91 & 0.92 & 0.97 & 0.95 \\
\hline T11 & 8.08 & 7.98 & 8.03 & 8.05 & 7.96 & 8.00 & 0.18 & 0.15 & 0.17 & 0.23 & 0.20 & 0.22 & 0.95 & 0.91 & 0.93 & 0.97 & 0.93 & 0.95 \\
\hline T12 & 8.17 & 8.01 & 8.09 & 7.98 & 7.91 & 7.94 & 0.21 & 0.18 & 0.20 & 0.22 & 0.19 & 0.21 & 0.92 & 0.89 & 0.91 & 0.99 & 0.82 & 0.91 \\
\hline T13 & 8.15 & 7.99 & 8.07 & 7.98 & 7.81 & 7.89 & 0.19 & 0.16 & 0.18 & 0.24 & 0.21 & 0.23 & 0.81 & 0.79 & 0.80 & 0.81 & 0.86 & 0.84 \\
\hline T14 & 8.19 & 8.03 & 8.11 & 7.95 & 7.93 & 7.94 & 0.18 & 0.14 & 0.16 & 0.21 & 0.17 & 0.19 & 0.83 & 0.78 & 0.81 & 0.85 & 0.91 & 0.88 \\
\hline T15 & 8.05 & 7.99 & 8.02 & 7.91 & 7.87 & 7.89 & 0.20 & 0.17 & 0.19 & 0.23 & 0.20 & 0.22 & 0.87 & 0.91 & 0.89 & 0.93 & 0.94 & 0.94 \\
\hline T16 & 7.89 & 7.76 & 7.82 & 7.85 & 7.73 & 7.79 & 0.23 & 0.19 & 0.21 & 0.27 & 0.23 & 0.25 & 0.86 & 0.87 & 0.87 & 0.92 & 0.89 & 0.91 \\
\hline T17 & 7.97 & 7.86 & 7.91 & 7.89 & 7.81 & 7.85 & 0.21 & 0.19 & 0.20 & 0.25 & 0.21 & 0.23 & 0.84 & 0.86 & 0.85 & 0.88 & 0.91 & 0.90 \\
\hline T18 & 7.99 & 7.89 & 7.94 & 7.91 & 7.83 & 7.87 & 0.23 & 0.18 & 0.21 & 0.26 & 0.22 & 0.24 & 0.81 & 0.85 & 0.83 & 0.83 & 0.88 & 0.86 \\
\hline T19 & 8.08 & 8.01 & 8.04 & 7.93 & 7.89 & 7.91 & 0.21 & 0.15 & 0.18 & 0.23 & 0.18 & 0.21 & 0.87 & 0.93 & 0.90 & 0.91 & 0.96 & 0.94 \\
\hline T20 & 7.96 & 7.87 & 7.91 & 7.90 & 7.81 & 7.85 & 0.22 & 0.19 & 0.21 & 0.25 & 0.20 & 0.23 & 0.81 & 0.83 & 0.82 & 0.86 & 0.85 & 0.86 \\
\hline T21 & 8.04 & 7.98 & 8.01 & 7.99 & 7.95 & 7.97 & 0.20 & 0.18 & 0.19 & 0.24 & 0.21 & 0.23 & 0.87 & 0.89 & 0.88 & 0.89 & 0.92 & 0.91 \\
\hline T22 & 8.13 & 8.06 & 8.09 & 8.01 & 7.97 & 7.99 & 0.18 & 0.16 & 0.17 & 0.21 & 0.19 & 0.20 & 0.88 & 0.91 & 0.90 & 0.91 & 0.93 & 0.92 \\
\hline T23 & 8.09 & 8.01 & 8.05 & 8.02 & 7.99 & 8.00 & 0.19 & 0.16 & 0.18 & 0.24 & 0.21 & 0.23 & 0.83 & 0.86 & 0.85 & 0.85 & 0.89 & 0.87 \\
\hline T24 & 8.13 & 8.11 & 8.12 & 7.98 & 7.93 & 7.95 & 0.21 & 0.19 & 0.20 & 0.23 & 0.19 & 0.21 & 0.85 & 0.89 & 0.87 & 0.91 & 0.95 & 0.93 \\
\hline T25 & 8.03 & 7.95 & 7.99 & 7.98 & 7.84 & 7.91 & 0.20 & 0.17 & 0.19 & 0.22 & 0.19 & 0.21 & 0.97 & 0.91 & 0.94 & 0.99 & 0.89 & 0.94 \\
\hline T26 & 8.13 & 7.99 & 8.06 & 8.11 & 7.97 & 8.04 & 0.19 & 0.17 & 0.18 & 0.21 & 0.18 & 0.20 & 0.83 & 0.78 & 0.81 & 0.85 & 0.87 & 0.86 \\
\hline T27 & 8.09 & 8.01 & 8.05 & 8.01 & 7.99 & 8.00 & 0.19 & 0.17 & 0.18 & 0.20 & 0.17 & 0.19 & 0.79 & 0.75 & 0.77 & 0.81 & 0.83 & 0.82 \\
\hline T28 & 8.07 & 7.97 & 8.02 & 8.01 & 7.89 & 7.95 & 0.18 & 0.14 & 0.16 & 0.20 & 0.13 & 0.17 & 0.78 & 0.81 & 0.80 & 0.83 & 0.85 & 0.84 \\
\hline T29 & 7.97 & 7.89 & 7.93 & 7.93 & 7.81 & 7.87 & 0.17 & 0.13 & 0.15 & 0.19 & 0.15 & 0.17 & 0.77 & 0.83 & 0.80 & 0.79 & 0.85 & 0.82 \\
\hline T30 & 8.07 & 8.01 & 8.04 & 8.05 & 7.99 & 8.02 & 0.15 & 0.13 & 0.14 & 0.17 & 0.13 & 0.15 & 0.76 & 0.79 & 0.78 & 0.79 & 0.82 & 0.81 \\
\hline Mean & & & 7.996 & & & 7.914 & & & 0.180 & & & 0.206 & & & 0.862 & & & 0.896 \\
\hline SEd & & & 0.028 & & & 0.030 & & & 0.009 & & & 0.008 & & & 0.030 & & & 0.041 \\
\hline $\operatorname{Cd}(0.05)$ & & & 0.057 & & & 0.062 & & & 0.018 & & & 0.016 & & & 0.061 & & & 0.080 \\
\hline
\end{tabular}


Table.3 Available Soil Nitrogen, Phosphorous and Potassium under different agroforestry practices

\begin{tabular}{|c|c|c|c|c|c|c|c|c|c|c|c|c|c|c|c|c|c|c|}
\hline \multirow[t]{3}{*}{ Treatment } & \multicolumn{6}{|c|}{$\mathbf{N}$} & \multicolumn{6}{|c|}{$\mathbf{P}$} & \multicolumn{6}{|c|}{$\mathbf{K}$} \\
\hline & \multicolumn{3}{|c|}{ Before } & \multicolumn{3}{|c|}{ After } & \multicolumn{3}{|c|}{ Before } & \multicolumn{3}{|c|}{ After } & \multicolumn{3}{|c|}{ Before } & \multicolumn{3}{|c|}{ After } \\
\hline & 30 & 60 & Mean & 30 & 60 & Mean & 30 & 60 & Mean & 30 & 60 & Mean & 30 & 60 & Mean & 30 & 60 & Mean \\
\hline T1 & 220.60 & 206.43 & 213.51 & 224.03 & 213.48 & 218.76 & 9.07 & 9.26 & 9.17 & 9.40 & 9.78 & 9.59 & 204 & 193 & 198.5 & 215 & 202 & 208.5 \\
\hline $\mathbf{T 2}$ & 319.44 & 269.51 & 294.47 & 330.79 & 290.68 & 310.74 & 10.59 & 10.37 & 10.48 & 10.76 & 10.68 & 10.72 & 217 & 205 & 211 & 227 & 214 & 220.5 \\
\hline T3 & 285.19 & 239.86 & 262.52 & 301.49 & 256.58 & 279.04 & 9.34 & 9.17 & 9.26 & 9.64 & 9.43 & 9.54 & 205 & 192 & 198.5 & 215 & 201 & 208 \\
\hline T4 & 358.84 & 351.37 & 355.1 & 379.08 & 365.30 & 372.19 & 10.54 & 9.60 & 10.07 & 10.80 & 10.17 & 10.49 & 214 & 198 & 206 & 224 & 207 & 215.5 \\
\hline T5 & 269.64 & 267.64 & 268.64 & 279.61 & 274.36 & 279.61 & 10.29 & 9.10 & 9.7 & 10.49 & 9.24 & 9.87 & 209 & 199 & 204 & 219 & 208 & 213.5 \\
\hline T6 & 207.03 & 179.51 & 193.27 & 207.74 & 228.54 & 218.14 & 9.06 & 8.50 & 8.78 & 9.18 & 8.66 & 8.92 & 198 & 190 & 194 & 207 & 198 & 202.5 \\
\hline T7 & 252.61 & 224.01 & 238.31 & 254.93 & 245.49 & 250.21 & 10.02 & 9.99 & 10.01 & 10.61 & 10.31 & 10.46 & 237 & 211 & 224 & 246 & 219 & 232.5 \\
\hline T8 & 226.41 & 194.39 & 210.4 & 230.98 & 199.69 & 215.21 & 9.28 & 9.11 & 9.2 & 9.37 & 9.31 & 9.34 & 211 & 202 & 206.5 & 220 & 210 & 215 \\
\hline T9 & 275.16 & 273.03 & 274.1 & 281.85 & 286.90 & 284.38 & 10.03 & 9.63 & 9.83 & 10.50 & 10.15 & 10.33 & 216 & 206 & 211 & 225 & 214 & 219.5 \\
\hline T10 & 208.87 & 197.04 & 202.96 & 221.65 & 206.69 & 214.17 & 10.03 & 9.61 & 9.82 & 10.17 & 9.68 & 9.93 & 201 & 191 & 196 & 210 & 199 & 204.5 \\
\hline T11 & 234.88 & 232.49 & 233.69 & 247.34 & 240.26 & 243.8 & 9.38 & 9.36 & 9.37 & 9.79 & 9.42 & 9.61 & 200 & 181 & 190.5 & 211 & 188 & 199.5 \\
\hline T12 & 289.57 & 262.68 & 276.13 & 294.92 & 272.73 & 283.3 & 10.41 & 10.18 & 10.3 & 10.78 & 10.37 & 10.58 & 231 & 204 & 217.5 & 242 & 211 & 226.5 \\
\hline T13 & 248.88 & 232.74 & 240.81 & 265.01 & 250.85 & 257.93 & 9.65 & 9.45 & 9.55 & 9.84 & 9.62 & 9.73 & 209 & 197 & 203 & 220 & 204 & 212 \\
\hline T14 & 350.80 & 310.94 & 330.87 & 358.00 & 327.57 & 342.79 & 10.35 & 10.22 & 10.29 & 10.46 & 10.40 & 10.43 & 211 & 199 & 205 & 222 & 206 & 214 \\
\hline T15 & 239.86 & 232.35 & 236.11 & 244.04 & 239.86 & 241.95 & 10.18 & 10.07 & 10.13 & 10.51 & 10.19 & 10.35 & 202 & 185 & 193.5 & 213 & 192 & 202.5 \\
\hline T16 & 207.26 & 210.64 & 208.95 & 210.53 & 216.87 & 213.7 & 8.56 & 8.16 & 8.36 & 8.71 & 8.51 & 8.61 & 199 & 188 & 193.5 & 210 & 196 & 203 \\
\hline T17 & 271.42 & 267.88 & 269.65 & 286.66 & 276.07 & 281.37 & 10.68 & 10.42 & 10.55 & 10.80 & 10.67 & 10.74 & 236 & 219 & 227.5 & 244 & 227 & 235.5 \\
\hline T18 & 242.73 & 226.57 & 234.65 & 258.70 & 244.67 & 251.69 & 8.54 & 7.92 & 8.23 & 8.81 & 8.02 & 8.42 & 206 & 195 & 200.5 & 214 & 203 & 208.5 \\
\hline T19 & 287.19 & 254.75 & 270.97 & 299.64 & 269.19 & 284.42 & 9.42 & 8.97 & 9.2 & 9.64 & 9.06 & 9.35 & 209 & 191 & 200 & 217 & 199 & 208 \\
\hline T20 & 193.41 & 214.33 & 203.87 & 202.09 & 223.88 & 212.99 & 9.74 & 8.91 & 9.33 & 9.78 & 9.22 & 9.5 & 204 & 190 & 197 & 212 & 198 & 205 \\
\hline T21 & 218.12 & 213.66 & 215.89 & 241.43 & 215.31 & 228.37 & 8.63 & 8.28 & 8.46 & 8.81 & 8.52 & 8.67 & 204 & 185 & 194.5 & 213 & 190 & 201.5 \\
\hline $\mathbf{T} 22$ & 248.30 & 243.05 & 245.68 & 261.22 & 251.96 & 256.59 & 10.56 & 9.99 & 10.2 & 10.68 & 10.17 & 10.43 & 240 & 221 & 230.5 & 249 & 226 & 237.5 \\
\hline T23 & 264.21 & 244.81 & 254.51 & 259.47 & 250.94 & 255.21 & 8.95 & 8.74 & 8.85 & 9.18 & 9.04 & 9.11 & 211 & 199 & 205 & 220 & 204 & 212 \\
\hline T24 & 305.35 & 292.46 & 298.91 & 310.85 & 308.57 & 309.71 & 10.19 & 10.11 & 10.15 & 10.44 & 10.24 & 10.34 & 217 & 203 & 210 & 226 & 208 & 217 \\
\hline T25 & 234.28 & 203.55 & 218.92 & 251.79 & 248.45 & 250.12 & 10.06 & 9.39 & 9.73 & 10.49 & 9.78 & 10.14 & 205 & 196 & 200.5 & 214 & 201 & 207.5 \\
\hline T26 & 194.23 & 187.89 & 191.06 & 212.82 & 208.21 & 210.52 & 8.31 & 7.98 & 8.15 & 8.66 & 8.03 & 8.35 & 193 & 184 & 188.5 & 200 & 189 & 194.5 \\
\hline T27 & 208.18 & 201.21 & 204.7 & 213.40 & 211.47 & 212.44 & 8.68 & 8.53 & 8.61 & 8.85 & 8.72 & 8.79 & 205 & 191 & 198 & 212 & 196 & 204 \\
\hline $\mathbf{T} 28$ & 213.24 & 207.90 & 210.57 & 222.16 & 216.12 & 219.14 & 8.52 & 7.65 & 8.09 & 8.74 & 7.88 & 8.31 & 201 & 183 & 192 & 208 & 188 & 198 \\
\hline T29 & 206.83 & 198.93 & 202.88 & 222.47 & 205.74 & 214.11 & 8.45 & 8.14 & 8.3 & 8.57 & 8.21 & 8.39 & 200 & 186 & 193 & 207 & 191 & 199 \\
\hline T30 & 218.07 & 216.78 & 217.43 & 228.91 & 221.64 & 225.28 & 8.74 & 8.52 & 8.63 & 8.92 & 8.78 & 8.85 & 199 & 177 & 188 & 206 & 182 & 194 \\
\hline Mean & & & 242.651 & & & 254.596 & & & 9.360 & & & 9.596 & & & 202.583 & & & 210.583 \\
\hline SEd & & & 11.112 & & & 10.709 & & & 0.217 & & & 0.246 & & & 3.510 & & & 3.822 \\
\hline $\operatorname{Cd}(0.05)$ & & & 22.727 & & & 21.904 & & & 0.443 & & & 0.504 & & & 7.180 & & & 7.818 \\
\hline
\end{tabular}


Table.4 Microbial populations under different agroforestry practices

\begin{tabular}{|c|c|c|c|c|c|c|c|c|c|c|c|c|c|c|c|c|c|c|}
\hline \multirow[t]{3}{*}{ Treatment } & \multicolumn{6}{|c|}{ Bacteria } & \multicolumn{6}{|c|}{ Fungi } & \multicolumn{6}{|c|}{ Actinomycets } \\
\hline & \multicolumn{3}{|c|}{ Before } & \multicolumn{3}{|c|}{ After } & \multicolumn{3}{|c|}{ Before } & \multicolumn{3}{|c|}{ After } & \multicolumn{3}{|c|}{ Before } & \multicolumn{3}{|c|}{ After } \\
\hline & 30 & 60 & Mean & 30 & 60 & Mean & 30 & 60 & Mean & 30 & 60 & Mean & 30 & 60 & Mean & 30 & 60 & Mean \\
\hline T1 & 19.89 & 17.57 & 18.73 & 22.02 & 19.56 & 20.79 & 10.38 & 10.04 & 10.21 & 12.41 & 11.89 & 12.15 & 17.15 & 15.27 & 16.21 & 19.25 & 17.16 & 18.21 \\
\hline $\mathbf{T 2}$ & 28.99 & 26.16 & 27.75 & 31.12 & 28.15 & 29.64 & 14.10 & 13.36 & 13.73 & 16.11 & 15.21 & 15.66 & 21.51 & 18.38 & 19.95 & 23.61 & 20.17 & 21.89 \\
\hline T3 & 26.23 & 24.65 & 25.44 & 28.36 & 26.64 & 27.5 & 12.65 & 12.05 & 12.35 & 14.66 & 13.9 & 14.28 & 20.11 & 18.81 & 19.46 & 22.21 & 20.6 & 21.41 \\
\hline T4 & 31.21 & 29.64 & 30.43 & 33.34 & 31.63 & 32.49 & 14.28 & 13.53 & 13.91 & 16.29 & 15.38 & 15.84 & 21.55 & 19.36 & 20.46 & 23.65 & 21.15 & 22.4 \\
\hline T5 & 23.77 & 21.67 & 22.72 & 25.90 & 23.66 & 24.78 & 11.47 & 10.47 & 10.97 & 13.48 & 12.32 & 12.9 & 19.74 & 17.98 & 18.86 & 21.84 & 19.77 & 20.81 \\
\hline T6 & 21.35 & 18.68 & 20.02 & 23.22 & 20.41 & 21.82 & 11.15 & 10.72 & 10.94 & 13.04 & 12.45 & 12.75 & 18.39 & 16.63 & 17.51 & 20.49 & 18.42 & 19.46 \\
\hline T7 & 29.18 & 28.90 & 29.04 & 31.05 & 30.63 & 30.84 & 15.13 & 13.52 & 14.33 & 17.04 & 15.25 & 16.15 & 21.02 & 20.88 & 20.95 & 23.12 & 22.67 & 22.9 \\
\hline T8 & 27.04 & 25.88 & 24.46 & 28.91 & 27.61 & 28.26 & 12.37 & 12.01 & 12.19 & 14.28 & 13.73 & 14.01 & 20.49 & 19.57 & 20.03 & 22.59 & 21.36 & 21.98 \\
\hline T9 & 32.32 & 30.29 & 31.31 & 34.19 & 32.02 & 33.11 & 15.34 & 13.94 & 14.64 & 17.23 & 15.67 & 16.45 & 22.66 & 21.63 & 22.15 & 24.76 & 23.42 & 24.09 \\
\hline T10 & 25.31 & 22.45 & 23.88 & 27.18 & 24.18 & 25.68 & 11.71 & 11.23 & 11.47 & 13.6 & 12.97 & 13.29 & 19.41 & 17.38 & 18.4 & 21.51 & 19.17 & 20.34 \\
\hline T11 & 21.26 & 19.70 & 20.48 & 23.37 & 21.61 & 22.49 & 11.51 & 10.02 & 10.77 & 13.29 & 11.71 & 12.5 & 17.32 & 16.4 & 16.86 & 19.42 & 18.19 & 18.81 \\
\hline T12 & 30.34 & 28.14 & 29.24 & 32.45 & 30.05 & 31.25 & 14.77 & 13.35 & 14.06 & 16.56 & 15.04 & 15.8 & 21.8 & 20.65 & 21.23 & 23.9 & 22.44 & 23.17 \\
\hline T13 & 27.23 & 26.18 & 26.71 & 29.34 & 28.09 & 28.72 & 12.25 & 11.84 & 12.05 & 14.05 & 13.53 & 13.79 & 20.84 & 18.34 & 19.59 & 22.94 & 20.13 & 21.54 \\
\hline T14 & 31.09 & 30.19 & 30.64 & 33.2 & 32.1 & 32.65 & 15.07 & 13.65 & 14.36 & 16.87 & 15.34 & 16.11 & 22.34 & 19.58 & 20.96 & 24.44 & 21.37 & 22.91 \\
\hline T15 & 25.97 & 23.46 & 24.72 & 28.08 & 25.37 & 26.73 & 12.08 & 10.18 & 11.13 & 13.86 & 11.87 & 12.87 & 20.73 & 17.84 & 19.29 & 22.83 & 19.63 & 21.23 \\
\hline T16 & 20.79 & 18.74 & 19.77 & 22.68 & 20.39 & 21.54 & 10.77 & 10.36 & 10.57 & 12.73 & 12.11 & 12.42 & 17.63 & 16.74 & 17.19 & 19.73 & 18.53 & 19.13 \\
\hline T17 & 29.43 & 25.96 & 27.7 & 31.32 & 27.61 & 29.47 & 14.54 & 13.33 & 13.94 & 16.5 & 15.01 & 15.76 & 21.02 & 20.57 & 20.8 & 23.12 & 22.36 & 22.74 \\
\hline T18 & 28.87 & 25.88 & 27.38 & 30.76 & 27.53 & 29.15 & 12.17 & 11.87 & 12.02 & 14.13 & 13.62 & 13.88 & 21.56 & 19.71 & 20.64 & 23.66 & 21.5 & 22.58 \\
\hline T19 & 31.58 & 28.20 & 29.89 & 33.47 & 29.85 & 31.66 & 14.70 & 13.76 & 14.23 & 16.66 & 15.41 & 16.04 & 22.03 & 21.07 & 21.55 & 24.13 & 22.86 & 23.5 \\
\hline T20 & 25.12 & 23.11 & 24.12 & 27.01 & 24.76 & 25.89 & 11.76 & 10.83 & 11.3 & 13.72 & 12.48 & 13.1 & 19.86 & 18.35 & 19.11 & 21.96 & 20.14 & 21.05 \\
\hline T21 & 20.31 & 18.04 & 19.18 & 22.09 & 19.89 & 20.99 & 10.23 & 9.32 & 9.78 & 11.66 & 10.91 & 11.29 & 17.61 & 16.9 & 17.26 & 19.71 & 18.69 & 19.2 \\
\hline T22 & 29.00 & 26.10 & 27.55 & 30.78 & 27.95 & 29.37 & 13.94 & 13.10 & 13.52 & 15.37 & 14.69 & 15.03 & 20.89 & 19.92 & 20.41 & 22.99 & 21.71 & 22.35 \\
\hline T23 & 27.80 & 24.87 & 26.34 & 29.58 & 26.72 & 28.15 & 12.37 & 11.78 & 12.08 & 13.8 & 13.37 & 13.59 & 19.97 & 18.37 & 19.17 & 22.07 & 20.16 & 21.12 \\
\hline T24 & 29.30 & 28.57 & 28.94 & 31.01 & 30.42 & 30.72 & 14.15 & 13.37 & 13.76 & 15.58 & 14.96 & 15.27 & 22.3 & 20.85 & 21.58 & 24.4 & 22.64 & 23.52 \\
\hline T25 & 24.66 & 22.43 & 23.55 & 26.44 & 24.28 & 25.36 & 10.84 & 10.39 & 10.62 & 12.27 & 11.98 & 12.13 & 19.89 & 18.58 & 19.24 & 21.99 & 20.37 & 21.18 \\
\hline T26 & 17.91 & 16.85 & 17.38 & 19.4 & 18.16 & 18.78 & 10.05 & 9.95 & 10 & 12.18 & 12.73 & 12.46 & 17.18 & 15.29 & 16.24 & 18.28 & 16.08 & 17.18 \\
\hline T27 & 26.14 & 25.54 & 25.84 & 27.63 & 26.85 & 27.24 & 11.55 & 11.08 & 11.32 & 13.31 & 13.92 & 13.62 & 20.61 & 18.57 & 19.59 & 22.71 & 20.36 & 21.54 \\
\hline T28 & 24.59 & 24.54 & 24.57 & 26.08 & 25.85 & 25.97 & 11.39 & 10.25 & 10.82 & 12.48 & 13.66 & 13.07 & 19.15 & 17.67 & 18.41 & 21.25 & 19.46 & 20.36 \\
\hline T29 & 28.29 & 26.30 & 27.3 & 29.78 & 27.61 & 28.7 & 11.77 & 11.44 & 11.61 & 13.67 & 13.94 & 13.81 & 21.42 & 19.4 & 20.41 & 23.52 & 21.19 & 22.36 \\
\hline T30 & 22.13 & 21.50 & 21.82 & 23.62 & 22.81 & 23.22 & 11.38 & 10.51 & 10.95 & 12.74 & 13.55 & 13.15 & 18.52 & 17.67 & 18.1 & 20.62 & 19.46 & 20.04 \\
\hline Mean & & & 25.230 & & & 27.099 & & & 12.121 & & & 13.972 & & & 19.387 & & & 21.300 \\
\hline SEd & & & 0.660 & & & 0.662 & & & 0.324 & & & 0.552 & & & 0.509 & & & 0.514 \\
\hline $\operatorname{Cd}(0.5)$ & & & 1.351 & & & 1.355 & & & 0.662 & & & 1.129 & & & 1.042 & & & 1.051 \\
\hline
\end{tabular}


Fig.1 Soil pH under different agroforestry practices

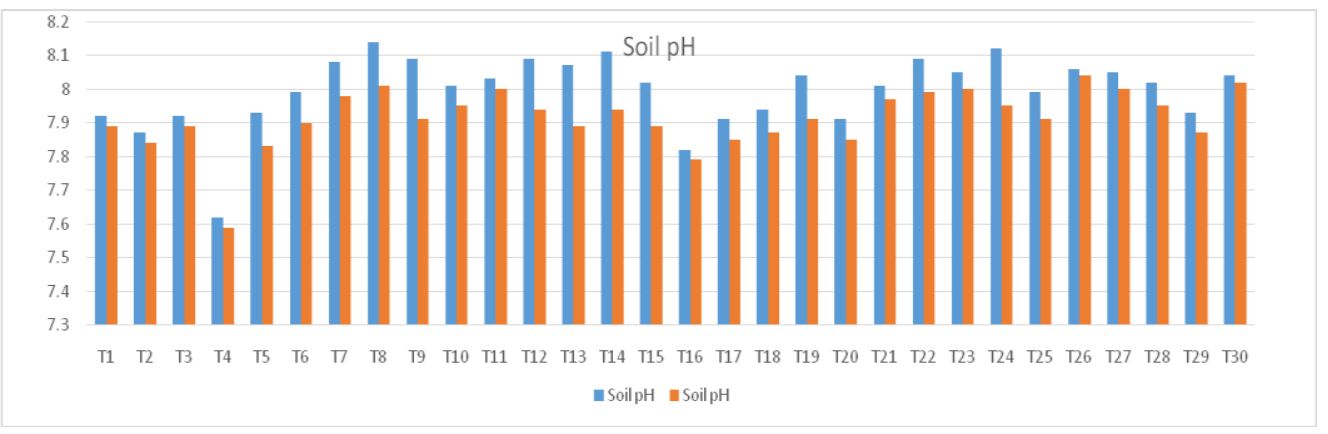

Fig.2 Soil EC under different agroforestry practices

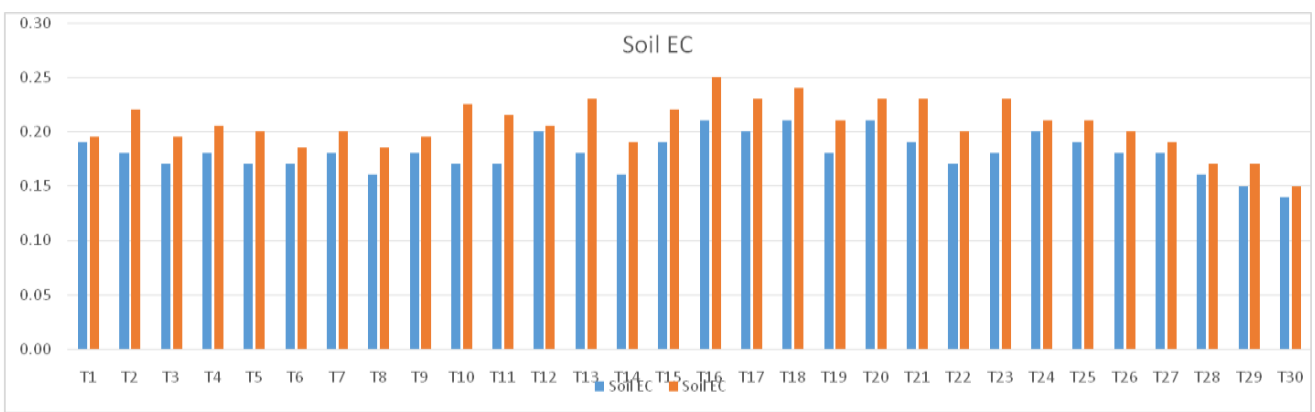

Figure 2. Soil EC under different agroforestry practices.

Fig.3 Soil OC under different agroforestry practices

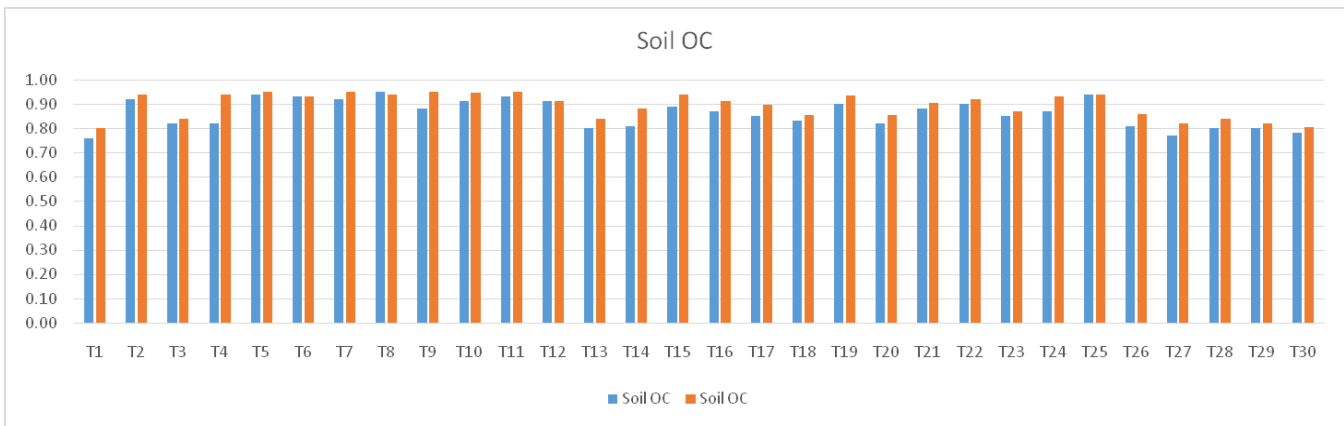

Fig.4 Available Nitrogen under different agroforestry practices

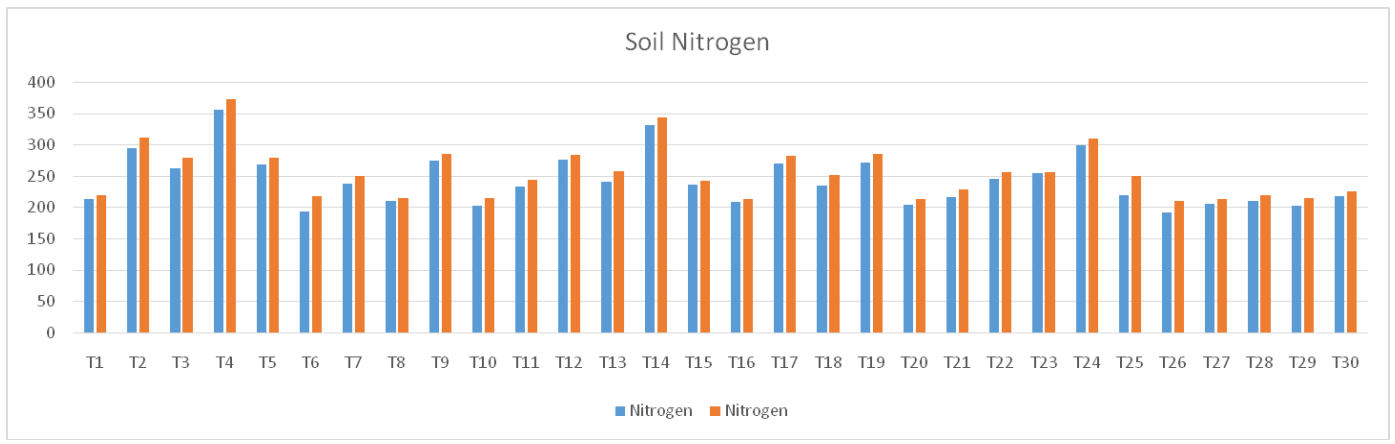


Fig.5 Available Phosphorus under different agroforestry practices

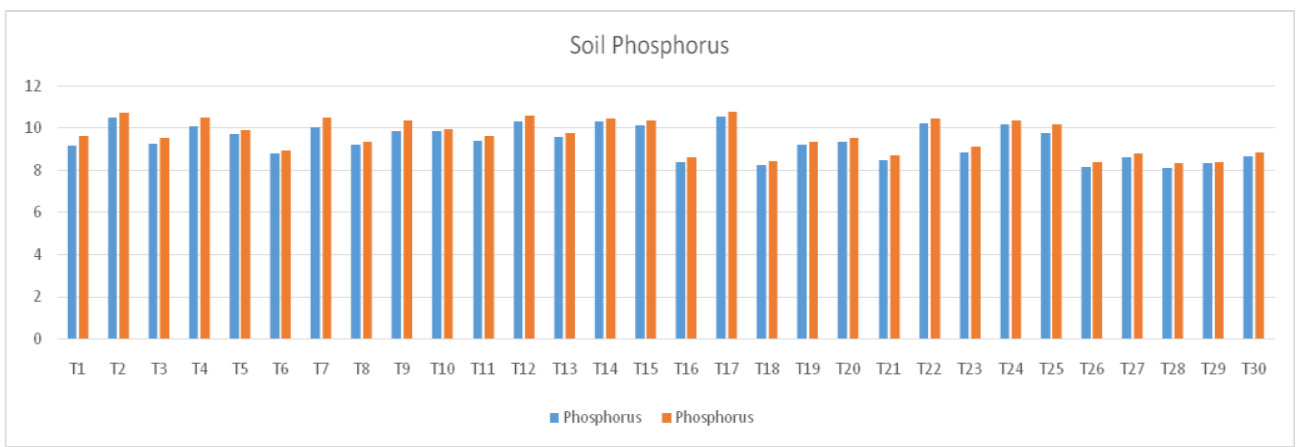

Fig.6 Available Phosphorus under different agroforestry practices

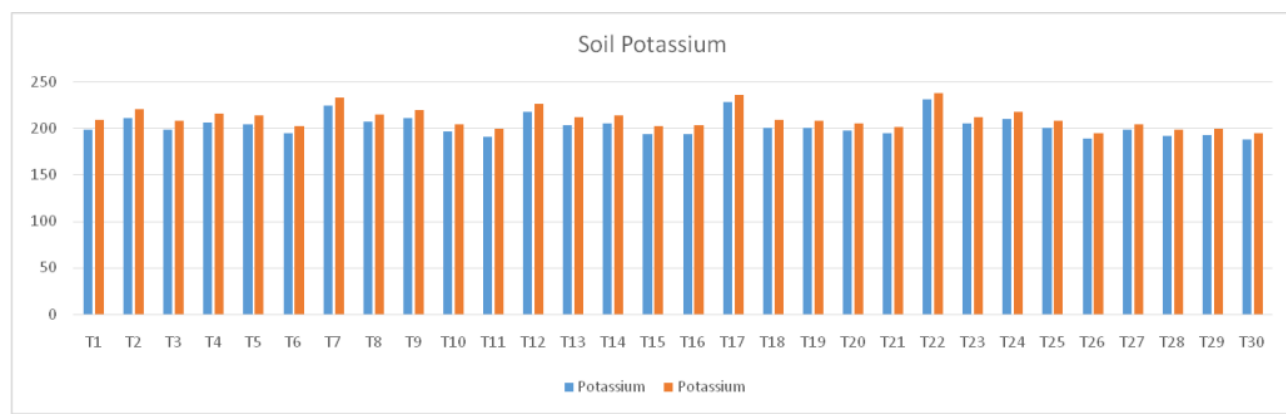

Fig.7 Soil Bacterial count under different agroforestry practices

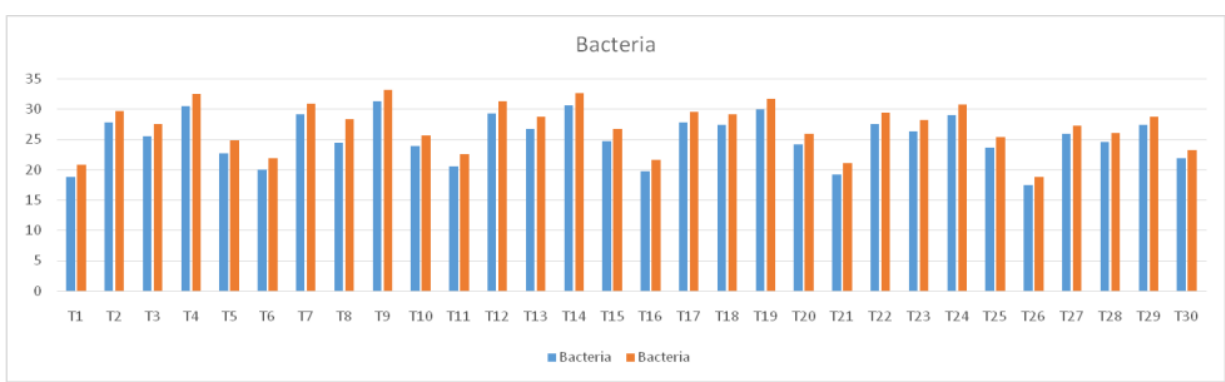

Fig.8 Soil Fungal population count under different agroforestry practices

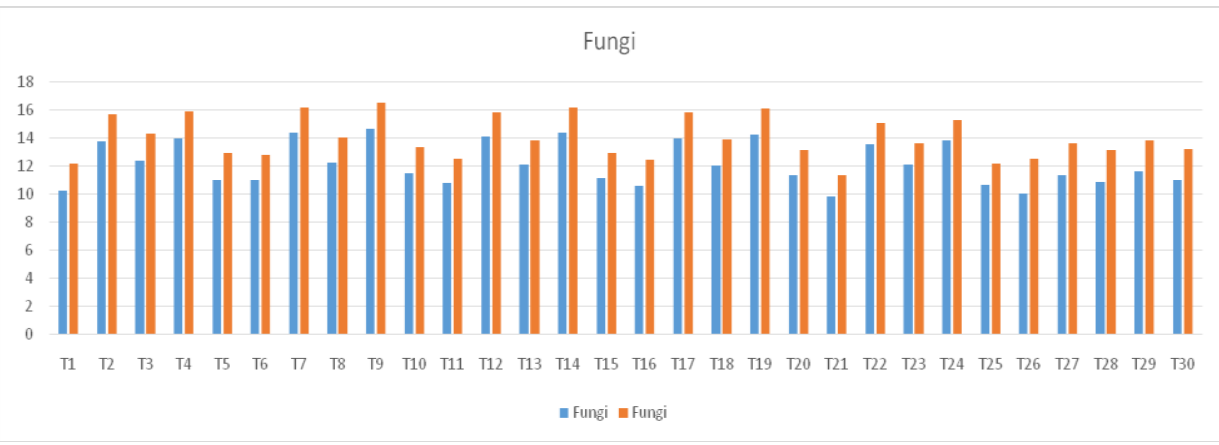


Fig.9 Soil actinomycetes population count under different agroforestry practices

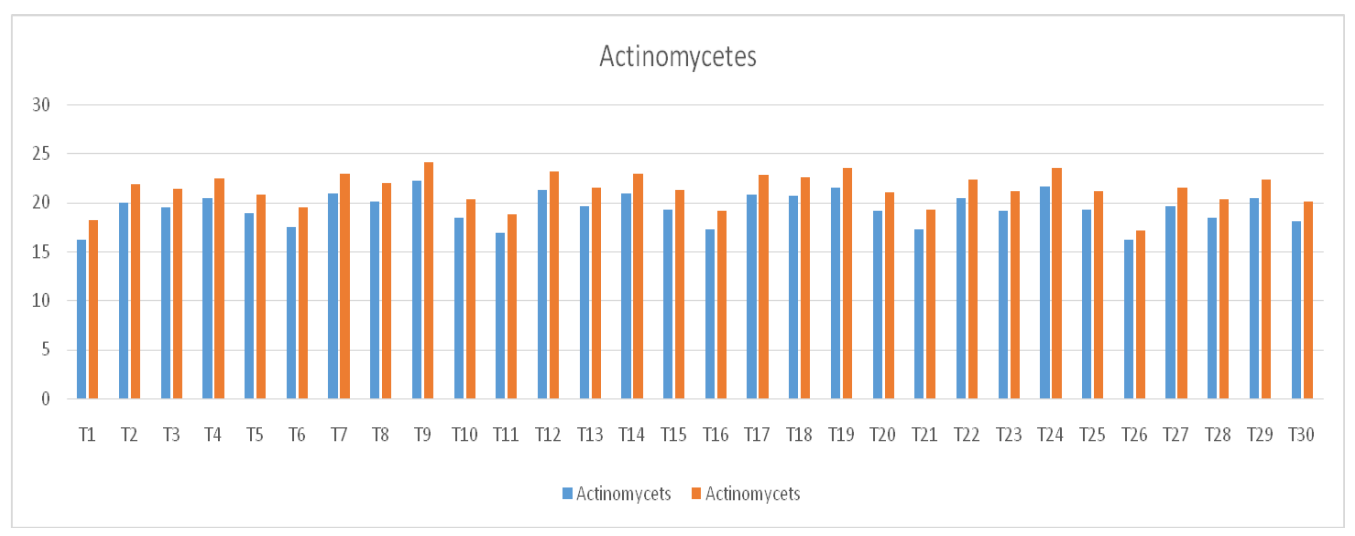

\section{Soil available phosphorus}

Data pertaining to the effect of intercrops on soil available phosphorus is presented in (Table 3). The highest available phosphorus of surface and sub-surface soil (10.48 and $10.72 \mathrm{~kg} \mathrm{ha}^{-1}$ ) was reported under green gram $\left(\mathrm{T}_{2}\right)$ and the lower amount of available phosphorus of surface and sub-surface soil ( 8.23 and $8.42 \mathrm{~kg} \mathrm{ha}^{-1}$ ) noticed under Populus deltoids + cowpea combination $\left(\mathrm{T}_{18}\right)$ (Table $3)$. Present findings are similar to the studies concluded by Mohan Raj (2004) improvement of available nitrogen, phosphorus and potassium under Simarouba based Agroforestry system. Jamaludheen (2010) reported that among the seven intercrops tried, the highest $\mathrm{P}$ content $\left(28.0 \mathrm{~kg} \mathrm{ha}^{-1}\right)$ was observed under groundnut intercropping and the lowest soil P under Gingelly $\left(17.4 \mathrm{~kg} \mathrm{ha}^{-1}\right)$ and small onion intercropping $\left(17.6 \mathrm{~kg} \mathrm{ha}^{-1}\right)$.

The tree alone treatment remained distinctively lowest available P content (13.7 $\mathrm{kg} \mathrm{ha}^{-1}$ ). Ashalatha (2011)reported that under Melia dubia based agroforestry system along the different tree - crop combinations, the highest soil available phosphorous $(24 \mathrm{Kg} \mathrm{ha}$ ${ }^{1}$ ) was recorded under groundnut followed by small onion intercropping $\left(23.0 \mathrm{Kg} \mathrm{ha}^{-1}\right)$ and the lowest was under tree alone treatment $\left(21.0 \mathrm{Kg} \mathrm{ha}^{-1}\right)$.

\section{Soil available potassium}

Effect of intercrops on soil available potassium is presented in (Table 3). Among the treatments, tree alone treatment had registered highest available $\mathrm{K}$ content of Casuarina equositifolia + green gram $\left(\mathrm{T}_{22}\right)$ of surface and sub-surface soil of 230.5 and $237.5 \mathrm{~kg} \mathrm{ha}^{-1}$ and the lowest available $\mathrm{K}$ of surface and sub-surface soil of was observed under Open area (Barren land) $\left(\mathrm{T}_{30}\right)$ (188 and $194 \mathrm{~kg} \mathrm{ha}^{-1}$ ) (Table 3). Similar result were reported that under Eucalyptus clone based agroforestry system, an increase over initial soil available potassium content $\left(192 \mathrm{~kg} \mathrm{ha}^{-1}\right)$ was observed in all the treatments including the pure tree crop in the post-harvest soil sample. Among the treatments, red gram intercropping had registered highest available $\mathrm{K}$ content of $204 \mathrm{~kg} \mathrm{ha}^{-1}$ followed by green gram $\left(201 \mathrm{~kg} \mathrm{ha}^{-1}\right)$ and the lowest available K was observed under tree alone treatment (196 $\mathrm{kg} \mathrm{ha}^{-1}$ ) (Selvam, 2011). Kathirvel (2003) also reported that the soil available potassium was improved under teak based agroforestry system.

\section{Microbial community}

\section{Bacteria}

Data with respect to effect of intercrops on soil bacterial population is presented in (Table 
4). An increase trend in the soil bacterial population was observed over the initial value in all the intercrops including tree alone treatment. However, the highest soil bacterial population (31.31 and $33.11 \times 10^{7}$ cfu's g $^{-1}$ soil) was reported under Melia dubia + blackgram combination $\left(\mathrm{T}_{9}\right)$ followed by Dalbergia sissoo + blackgram combination $\left(\mathrm{T}_{14}\right)$ (30.64 and $\left.32.65 \times 10^{7} \mathrm{cfu}^{\prime} \mathrm{s} \mathrm{g}^{-1}\right)$ and the lower amount of soil bacterial population (26.99 and 24.87 x $10^{7}$ cfu's g $^{-1}$ ) noticed under Khaya senegalensis tree alone $\left(\mathrm{T}_{1}\right)$ (Table 4). Present findings are similar to the studies concluded by, Meshram et al., (2016)observed that the maximum population of bacterial (26.28 CFU X $\left.10^{7} \mathrm{~g}\right)$ was recorded under $A$. mangium, the maximum population of fungal $\left(15.90 \mathrm{CFU} \mathrm{X} 10^{4} \mathrm{~g}\right)$ was recorded under A. holosericea and maximum population of actinomycetes (14.27 CFU X $10^{6} \mathrm{~g}$ ) under $G$. sepium in ten different $\mathrm{N}$ fixing tree species viz., Cassia siamea, Acacia catechu, Acacia mangium, Gliricidia sepium, Pterocarpus marsupium, Acacia holosericea, Acacia auriculiformis, Albizzia lebbeck, Casuarina equisetifolia and Dalbergia sissoo..

\section{Fungi}

Data pertaining to effect of intercrops on soil fungal population is presented in (Table 4). There was an increase trend of fungal population in the soil after intercropping under all the treatments including tree alone treatment when compared to initial value. The highest amount of fungal population of surface and sub-surface soil was observed under Melia dubia + blackgram combination $\left(\mathrm{T}_{9}\right)\left(14.64\right.$ and $16.45 \times 10^{5} \mathrm{cfu}^{\prime} \mathrm{g}^{-1}$ soil) followed by Dalbergia sissoo + blackgram combination $\left(\mathrm{T}_{14}\right)\left(14.36\right.$ and $16.11 \times 10^{5}$ cfu's $g^{-1}$ soil) treatments and the lowest fungal population of surface and sub-surface soil was observed in Khaya senegalensis tree alone ( $\left.\mathrm{T}_{1}\right)\left(10.21\right.$ and $12.15 \times 10^{5} \mathrm{cfu}^{\prime} \mathrm{g}^{-1}$ soil $)$
(Table 4). The positive interaction of both soil and crop engaged in agroforestry are reflected to improve the action of soil fauna(Kang et al., 1985). Ravi (2005)reported that the microbial population under 15 year old Ailanthus excelsa was $56.0 \times 10^{7} \mathrm{cfu} \mathrm{s} \mathrm{g}^{-1}$ soil bacteria, $24.0 \times 10^{5} \mathrm{cfu}^{\prime} \mathrm{s} \mathrm{g}^{-1}$ soil fungi and $32.0 \times 10^{2}$ cfu's $\mathrm{g}^{-1}$ soil actinomycetes respectively.

\section{Actinomycetes}

Data on to effect of intercrops on soil actinomycetes population is presented in (Table 4). An increase trend in thesoil actinomycetes population was observed over the initial value in all the intercrops including tree alone treatment. However, the highest soil actinomycetes population $(22.15$ and 23.09x $10^{2}$ cfu's $\mathrm{g}^{-1}$ ) was reported under Melia dubia + blackgram combination $\left(\mathrm{T}_{9}\right)$ followed by Casuarina equositifolia + blackgram $\left(\mathrm{T}_{24}\right)\left(21.58\right.$ and $23.52 \times 10^{2}$ cfu's $\mathrm{g}^{-1}$ ) and the lower amount of soil actinomycetes population (16.21 and 18.21x $10^{2}$ cfu's $\mathrm{g}^{-1}$ ) noticed under Khaya senegalensis tree alone $\left(\mathrm{T}_{1}\right)$ (Table 4$)$. Ravi (2005)reported that the microbial population under 15 year old Ailanthus excelsa was 56.0 x $10^{7} \mathrm{cfu}^{\prime} \mathrm{g}^{-1}$ soil bacteria, $24.0 \times 10^{5} \mathrm{cfu}$ 's $\mathrm{g}^{-1}$ soil fungi and $32.0 \times 10^{2} \mathrm{cfu}^{\prime} \mathrm{g}^{-1}$ soil actinomycetes respectively.

In conclusion soil fertility and microbial community are increased under different treecrop combination. These type of tree-crop combination has a significant bearing on these parameters in soil and hence having a choice of tree crop combination for better microbial activity in soil.There was decrease trend on the soil $\mathrm{pH}$ by the intercrops. An increasing trend of EC, soil organic carbon, available soil nitrogen, available soil phosphorus, and available soil potassium and microbial population. Amongst tree crop combination under investigation Melia dubia + blackgram 
combination $\left(\mathrm{T}_{9}\right)$ land uses has significantly higher soil fertility status and microbial activities. The lowest EC, soil organic carbon, available soil nitrogen, available soil phosphorus, and available soil potassium was observed under Khaya senegalensis trees alone $\left(\mathrm{T}_{1}\right)$.

\section{Acknowledgments}

The authors wish to acknowledge the help and support rendered by Head, Department of Agroforestry and Dean (Forestry), forgranting the permission to conduct the research in Forest College and Research Institute, Mettupalayam. Author is also thankful to all department staff and lab assistant those who all help during the research work. Authors acknowledge the anonymous reviewers for prompt and critical review and suggestions to improve the manuscript.

\section{References}

Ashalatha A. 2011. Development of suitable Melia dubia based agroforestry system for higher productivity and carbon sequestration.M.Sc. Thesis, Tamil Nadu Agricultural University, Coimbatore. .

Baisden W Troy, and Roger L Parfitt. 2007. Bomb 14 C enrichment indicates decadal $\mathrm{C}$ pool in deep soil? Biogeochemistry 85 (1):59-68.

Dhyani SK, and DS Chauhan. 1990. Nitrogen fixing trees suitable for agro-forestry in Meghalaya. Indian Journal of Hill Farming 3 (2):65-68.

Dollinger Jeanne, and Shibu Jose. 2018. Agroforestry for soil health. Agroforestry systems 92 (2):213-219.

Goberna M, J Sánchez, JA Pascual, and C García. 2006. Surface and subsurface organic carbon, microbial biomass and activity in a forest soil sequence. Soil Biology and Biochemistry 38 (8):2233-
2243.

Gupta Raj Kumar, Vijay Kumar, KR Sharma, Tejbir Singh Buttar, Gobinder Singh, and Gowhar Mir. 2017. Carbon sequestration potential through agroforestry: A review. International Journal of Current Microbiology and Applied Science 6 (8):211-220.

Jackson ML. 1973. Methods of chemical analysis. Prentic Hall., EngleWood Cliffs, NTJ.

Jamaludheen V. 2010. Studies on intercropping with Casuarina junghuhniana at different stand densities in western zone of TamilNadu. PhD. Thesis, Tamil Nadu Agricultural University, Coimbatore.

Jobbágy Esteban G, and Robert B Jackson. 2000. The vertical distribution of soil organic carbon and its relation to climate and vegetation. Ecological applications 10 (2): 423-436.

Kang BT, H Grimme, and TL Lawson. 1985. Alley cropping sequentially cropped maize and cowpea with Leucaena on a sandy soil in Southern Nigeria. Plant and soil 85 (2):267-277.

Kathirvel P.. 2003. Studies on compatibility of forage crops under established teak (Tectona grandis Linn.) plantation.. M.Sc. Thesis, Tamil Nadu Agricultural University, Coimbatore. .

Kikon Grace, DR Bhardwaj, and CL Thakur. 2017. Turmeric cultivation under bamboo based agroforestry systemEffect of bamboo species and mulch material on weeds and soil characteristics. Indian Journal of Agroforestry 19 (2):65-71.

Lal R. 1989. Agroforestry systems and soil surface management of a tropical alfisol. Agroforestry systems 8 (2):97111.

Meshram Nandkishor, Vijay Dalvi, Ashish Shigwan, and Satish Narkhade. 2016. Microbial community and soil fertility 
status as influenced by different nitrogen fixing tree species in alfisol. Indian Journal of Agroforestry 18 (2):53-58.

Mohan Raj T.. 2004. Studies on compatibility of agricultural crops with Simarouba glauca DC. M.Sc. Thesis, Tamil Nadu Agricultural University, Coimbatore.

Mutanal SM, AS Prabhakar, and BS Nadagoudar. 2000. Groundnut (Arachis hypogaea-teak (Tectona grandis) interaction in agroforestry system. Indian Journal of Agricultural Sciences 70 (7):463-465.

Nair PKR. 1984. Soil productivity aspects of agroforestry. Vol. 1: International Council for Research in Agroforestry Nairobi, Kenya.

Nair PKR. 1989. Agroforestry defined. Dalam. Agroforestry Systems in the Tropics, Kluwer Academic in Cooperation With ICRAF, Nairobi. Kenya.

Nair PKR. 2011. Agroforestry systems and environmental quality: introduction. Journal of environmental quality 40 (3):784-790.

Olsen Sterling Robertson. 1954. Estimation of available phosphorus in soils by extraction with sodium bicarbonate: US Department of Agriculture.

Palani M. 1996. Studies on intercropping agricultural crops with multiutility trees under rainfed condition. Ph.D. Thesis, Tamil Nadu Agricultural University, Coimbatore. .

Panse Vinayak Govind, and Pandurang Vasudeo Sukhatme. 1954. Statistical methods for agricultural workers. Statistical methods for agricultural workers.

Parkinson Dennis, Timothy RG Gray, and Stanley Thomas Williams. 1971. Methods for study-ing the ecology of soil micro-organisms. Methods for study-ing the ecology of soil micro- organisms.

Portella Cibele Mascioli Rebello, Maria de Fátima Guimarães, Christian Feller, Inês Cristina de Batista Fonseca, and João Tavares Filho. 2012. Soil aggregation under different management systems. Revista Brasileira de Ciência do Solo 36 (6):1868-1877.

Prasad, R, R Newaj, N.K Saroj, V.D Tripathi, and S.K Dhyani. 2015. Pattern of soil organic carbon decomposition in some agroforestry systems in Bundelkhand region of Central India. Indian Journal of Agroforestry 17 (2):18-21.

Ravi. 2005. Investigation on shade tolerant fodder crops for Ailanthus based agroforesty system.. M.Sc. Thesis, Tamil Nadu Agricultural University, Coimbatore. .

Selvam. 2011. Evaluation of suitable intercrops for Eucalyptus clone based Agroforestry system. M.Sc (Forestry), Tamil Nadu Agricultural University, Coimbatore.

Sharma, N.K., Ranjan, Rajeev, Pramanik, Monalisha, and N.M Alam. 2016. Dynamics of soil physical and chemical properties under wheat (Triticum aestivum L.)-poplar (Populus deltoides M.) based agroforestry system. Indian Journal of Agroforestry 18 (2):41-46.

Singh, Rambir, Singh, Charan, and Ajay Gulati. 2018. Growth and Yield Attributes of Melia composita and Emblica officinalis Based Agri-SilviMedicinal Agroforestry in Degraded Lands. Indian Forester 144 (1):78-83.

Stanford George, and Leah English. 1949. Use of the flame photometer in rapid soil tests for $\mathrm{K}$ and $\mathrm{Ca}$. Agronomy Journal 41 (9):446-447.

Subbiah B, and GL Asija. 1956. Alkaline permanganate method of available nitrogen determination. Current Science 25:259. 
Veldkamp Edzo, Anja Becker, Luitgard Schwendenmann, Deborah A Clark, and Hubert Schulte-Bisping. 2003. Substantial labile carbon stocks and microbial activity in deeply weathered soils below a tropical wet forest. Global change biology 9 (8):1171-1184.

Walkley Aldous, and I Armstrong Black. 1934. An examination of the Degtjareff method for determining soil organic matter, and a proposed modification of the chromic acid titration method. Soil science 37 (1):29-38.

Willison T. W., Splatt, P. R., \& Anderson, J.
M.. 1990. Nutrient loading of a forest soil.. Oecologia 82 (4):507-512.

Yadav R. S., Yadav, B. L., and Chhipa, B. R. 2008. Litter dynamics and soil properties under different tree species in a semi-arid region of Rajasthan, India. Agroforestry systems 73 (1):1-12.

Young A.. 1989a. 10 hypotheses for soilagroforestry research Agroforestry Today 1:13-17.

Young A.. 1989b. Agroforestry for Soil Conservation: $\mathrm{CAB}$ International.

\section{How to cite this article:}

Baswant Sushant Pralhad, P. Rajendran, M. P. Divya, R. Rajeswari, G. Thangamani and Ramaha, C. 2020. Assessing the Effect of Different Agroforestry Practices on Soil Physicochemical Properties and Microbial Activity. Int.J.Curr.Microbiol.App.Sci. 9(12): 2802-2816. doi: https://doi.org/10.20546/ijcmas.2020.912.335 